\title{
P53-Mdm2 Loop Stability and Oscillatory Dynamics with Mdm2-Induced Delay Effect in P53
}

\author{
Mohd Younus Baba, Mohammad Saleem and Abdur Raheem
}

\begin{abstract}
In this paper, we consider P53-Mdm2 negative feedback loop supposed to be the core circuit of genome. We study stability and the oscillatory dynamics of the loop. Many of the studies modeled this loop by delay-differential equations with P53-induced transcriptional delay in the production of Mdm2. We, however, highlight the importance of Mdm2induced delay in the degradation of P53 protein. We consider two forms of P53 protein i.e., plain P53 and active P53 along with its principal antagonist protein Mdm2 to formulate a minimal model. Active P53 finds its inclusion in the loop in the presence of DNA damage represented by a Boolean variable ' $s$ '. The analysis of the model provides thresholds on delays using Nyquist criterion such that delays in the degradation of P53 lower than these thresholds guarantee stability of the loop in that all proteins plain P53, active P53 and Mdm2 approach to stable equilibrium state. The oscillatory dynamics in proteins, if any, would exist beyond these thresholds.
\end{abstract}

\section{Introduction}

Protein P53 is a well-known tumor suppressor. Also known as the "Master of the Genome", it helps preserve genomic integrity in mammalian cells [1,2]. About 50 percent of human cancers occur due to mutations in the P53 gene [3]. In cells, P53 stays in association with another protein Mdm2 in an interesting relationship: P53 $\rightarrow$ Mdm2 $\dashv$ P53 i.e., P53 promotes transcription of $\mathrm{Mdm} 2$ and the Mdm2 on the other hand stimulates degradation of P53. This relationship, known since early 90s, is called the P53-Mdm2 negative feedback loop. It helps maintain P53 levels low in normal cells $[1,4,5,6,7]$. When the cells are stressed due to DNA damage (double strand breaks, single strand breaks) or oncogenic activation, the negative feedback loop with Mdm2 is disrupted (or weakened) and P53 no longer in strict control of Mdm2 starts accumulating and triggers a transcriptional program that may lead to cell senescence, DNA damage repair or apoptosis $[2,8]$. It is then not surprising that $\mathrm{P} 53$ activity is restricted by $\mathrm{Mdm} 2$ in normal cells and its concentration is kept low in these cells $[1,9]$.

2010 Mathematics Subject Classification. 92B05; 92C37; 92C42; 92C50.

Key words and phrases. P53, Mdm2, DDE, Time delays, Stability, Oscillations.

Corresponding author: Mohd Younus Baba. 
The main characteristic feature of the P53-Mdm2 regulatory network has been its oscillatory behavior both in stressed and non-stressed conditions. The generation of oscillations in this network has been a challenge to modelers, more so, because they have been well aware of the fact that a negative feedback is necessary for oscillations but it is not sufficient [10]. It can be argued that a negative feedback with only two elements i.e., P53 $\rightarrow \mathrm{Mdm} 2 \dashv$ P53 cannot oscillate [11].

The occurrence of oscillations in the P53 concentration after radiation induced DNA damage has been reported in many experimental studies $[1,12,13]$. The earlier works consider cell populations and show occurrence of one or two irregularly spaced P53 peaks with different amplitudes. Alon and collaborators, in their important studies on single cells in culture under gammairradiated stressed conditions give well-established oscillation's pattern in the form of a series of discrete pulses in both P53 and Mdm2 concentrations [13, 14]. In the first study [13], this group reports a sort of "digital" response to stress with peaks of gene expression of constant amplitude repeating in average a number of times proportional to signal strength. In the second study [14], this team presents a more regular oscillatory pattern during the whole observation time (up to 3 days) and in large traction of cells proportional to the radiation dose. Theoretical studies suggest that oscillations can arise from a combination of positive and negative feedbacks $[11,15]$ or from a long negative feedback by augmenting the core P53-Mdm2 loop with some putative intermediary (or unidentified supplementary) components $[16,17]$. Some later experiments show that P53 pulses occur due to repeated initiation of ATM activation from persistent DNA damage and that P53 pulses are excitable [18]. Excitability means once a pulse is initiated it will make a full pulse irrespective of whether the damage signal persists or not. Experimental study of Batchelor et al. [19] suggests that it requires a positive feedback loop in the P53-Mdm2 network to generate excitable P53 pulses.

Mathematical modeling has played a very important role in exploring challenging problems in biological, medical and physical sciences [3, 20,21, 22]. The contribution of modeling has been in producing analytical and numerical solutions of many difficult real world problems. It has dealt with questions of stability of solutions including exploring even regions of chaotic nature. In the studies on cancer, modeling in many cases has validated experimental data on oscillatory behavior in P53 and Mdm2 concentrations (see references listed above). Mihalas et al. [23] consider the interaction of $\mathrm{P} 53$ and Mdm2 genes in a chemical kinetics-type model of four differential equations: two equations for mRNAs and other two equations for proteins. Through computer simulation of this model, they show the oscillatory behavior with some realistic assumptions on the model parameters. Bar-Or et al. [16] consider a long negative feedback loop for possible oscillations by adding an intermediary component in between P53 and Mdm2 and thus introducing the idea of delay between P53 activation and P53-dependent induction of Mdm2. Mihalas et al. [24] modify the model considered in Mihalas et al. [23] and introduce three stiff delays $\tau_{i}$ in it: $\tau_{1}$ is the delay 
that represents the total time required to complete the three activities namely (i) binding of Mdm2 to P53 (ii) interactions of the Mdm2 isoforms (P76 and P90) and (iii) translocation of P53 in cytosol. $\tau_{2}$ is the delay required for P53 to enter in the nucleus to bind P2 promoter of the Mdm2 gene; $\tau_{3}$ is the delay required for the HAUSP to interact with P53 and Mdm2 to de-ubiquitinate both proteins. Mihalas et al. [24] obtain oscillatory behavior similar to that observed experimentally $[13,16]$. Zatorsky et al. [14] consider a set of six models covering almost all the variations in molecular interactions, loops, non-linearities, inclusion of stiff-delays etc. of previous models. They simulate all the six models numerically and produce onset of oscillations. Using the idea of the model III of Zatorsky et al. [14], Bottani and Grammaticos [25] study a time delayed differential equation model of the regulatory loop for P53 expression. Their model, based on a "minimal" set of well-established bio-molecular regulations, shows how a negative feedback loop with delay can be used in dual role i.e., to maintain a stable steady state and to generate oscillations depending on the input signal. In Layek et al. [26], authors give a general theoretical framework to convert genetic pathways information to Boolean networks to include multivariate interactions between the genes. Using this approach to the P53 pathway including ATM and Wip1 considered in $[13,18]$ and simulating their resulting Boolean network, Layek et al. [26] exhibit P53 related oscillations similar to those reported in [13, 18]. Using a computational model, Purvis et al. [27] identify a sequence of precisely timed drug addition of small molecule Nutlin-3 that could alter P53 pulses (due to double strand breaks) to sustained P53 response. They report different cell-fate decisions: recovery of cells from DNA damage with P53 pulses and cell cycle arrest (senescence) with sustained P53 response. Sun et al. [28] analyze a delay-differential equation model consisting of 12 equations including 4 equations for $\mathrm{p} 53$, mdm2, wip 1 and $\mathrm{p} 21 \mathrm{mRNAs}$ and rest for proteins. They include phosphorylation, acetylation of proteins and dynamic activation of ATM into their model. Their results relate to the basal dynamics of P53 and show that the dynamics of P53 under non-stressed conditions is initiated by an excitable mechanism and cells fully respond when they are confronted with severe damage. Chong et al. [29] extend the model of Sun et al. [28] by incorporating into it some recently found molecular interactions and hypotheses and thus invoking more accurate design principles of the P53 molecular system. In particular, they add the dynamics of MdmX (an Mdm2-related P53 antagonist protein) along with P53-Mdm2, Mdm2-MdmX and P53-MdmX complexes. Their model results very well exhibit the basal dynamics particularly the excitability response and oscillatory behavior of $\mathrm{P} 53$ proteins.

The study of this paper concerns stability and the oscillatory dynamics of the circuit for P53 expression but in a different context. In most of the delay-differential equation models formulated for the P53-Mdm2 regulatory loop, the production of Mdm2 has been considered to be affected by P53-induced transcriptional delays. In a recent paper, Pant and Lozano [30], while working on a genetically engineered mouse model, observe delayed P53 degradation through Mdm2 via Mdm4 after DNA damage. Moreover, different proteins in the family of Mdm2 such as Mdm4 
[30], MdmX [29], and P76Mdm2, P90Mdm2 [24] have been suggested to have taken part in the degradation of P53. We postulate that the degradation of P53 by any of these proteins along with $\mathrm{Mdm} 2$ may not be simultaneous. In this paper, we therefore incorporate Mdm2-induced timedelay degradation in P53. This delay represents the time required for Mdm2 to bind P53 and the time required for the linkage between $\mathrm{Mdm} 2$ and any of its isoforms. The main question that this paper addresses to is whether a Mdm2-induced delay in P53 degradation can explain previously observed oscillations in P53 concentration in absence or presence of stress?

\section{The model}

Bottani and Grammaticos [25] consider a minimal model for the P53-Mdm2 regulatory loop

$$
\begin{aligned}
& \frac{d x}{d t}=a-s x-b x-c x z \\
& \frac{d y}{d t}=s x-b y-d y z \\
& \frac{d z}{d t}=h+f F\left(x\left(t-\tau_{3}\right)+y\left(t-\tau_{3}\right)\right)-g(s) z .
\end{aligned}
$$

This model has two types of variables i.e. state variables $x(t), y(t), z(t)$ for plain P53, active P53 and $\mathrm{Mdm} 2$ protein populations at time $t$ and binary Boolean variable $s$ which assumes value 1 (one) when the system is stressed and 0 (zero) when the system is not stressed.

Further interpretation of functions and model parameters are given below.

$a$ - is the constant production rate of plain P53 (x)

$b$ - denotes self degradation rate of both plain P53 (x) and active P53 ( $y)$

$c$ and $d$ - are the parameters that represent different induced degradation rates of plain P53 $(x)$ and active P53 $(y)$ resulting from their different binding strengths with Mdm2. Based on experimental data, it is assumed that $d<c$.

$h$ - is the basal production rate of Mdm2 protein

$f$ - is the P53-induced production rate of $\operatorname{Mdm} 2(z)$

$F$ - defines the process how the transcription induced by P53 for the production of Mdm2 works. $F$ is assumed to have a form of Hill function as follows:

$$
F(u)=\frac{u^{4}}{K^{4}+u^{4}}
$$

with $K$ representing the dissociation constant between $\mathrm{P} 53$ and the Mdm2 promoter $g$ - is a bi-valued function of Boolean variable $s$ defined as

$$
g(s)=(1-s) g_{1}+s g_{2}
$$


with $g_{1}$ and $g_{2}$ as positive constants. $g_{1}$ represents the self-degradation rate of Mdm2 when $s=0$ i.e., when the system is not stressed. On the other hand $g_{2}$ denotes the self-degradation rate of $\operatorname{Mdm} 2$ for $s=1$ i.e., when the system is stressed due to DNA damage. It is assumed that $g_{1}<g_{2}$. $\tau_{3}$ - represents the time-delay in the production of $\mathrm{Mdm} 2$ protein $(z)$ after transcription initiation induced by P53.

Model (2.1) represents a negative feedback loop of P53-Mdm2 interactions, where P53 protein stimulates $\mathrm{Mdm} 2$ protein and Mdm2 degrades $\mathrm{P} 53$ protein. It is well known that the ubiquitination of $\mathrm{P} 53$ is a must for its degradation. Mdm2 ubiquitinates P53 through its ring domain in the C-terminus. Mihalas et al. [24] consider two Mdm2 proteins namely P90Mdm2 and P76Mdm2 and their interaction within to complete the ubiquitination of P53 by Mdm2. They suggest that while P53 stimulates P76Mdm2 for its production but it having a truncated $\mathrm{N}$-terminus does not bind $\mathrm{P} 53$ and hence cannot ubiquitinate it. It is indeed $\mathrm{P} 90 \mathrm{Mdm} 2$ protein having complete $\mathrm{N}$ terminus that binds P53 and ubiquitinates it through a link of P76Mdm2 in between P53 and P90Mdm2. Mihalas et al. [24] introduce stiff time-delay $\tau_{1}$ in Mdm2 protein and use the term $y_{2}\left(t-\tau_{1}\right)$ in their model to represent the time delay required for Mdm2 to bind P53 and for completion of the loop. Recently, Pant and Lozano [30] reported Mdm2-induced time-delayed degradation of P53 while working on a genetically engineered mouse model. Motivated by these studies, we assume in this paper that $\tau_{1}$ and $\tau_{2}$ are time delays that consist of (i) time required for Mdm2 to bind plain P53 $(x)$ and active P53 (y) respectively and (ii) the time required for the linkage of $\mathrm{Mdm} 2$ with other E3 ligases or its isoforms to complete the degradation process. Incorporating these assumptions, the model (2.1) is modified as

$$
\begin{aligned}
& \frac{d x}{d t}=a-s x-b x-c x z\left(t-\tau_{1}\right), \\
& \frac{d y}{d t}=s x-b y-d y z\left(t-\tau_{2}\right), \\
& \frac{d z}{d t}=h+f F\left(x\left(t-\tau_{3}\right)+y\left(t-\tau_{3}\right)\right)-g(s) z,
\end{aligned}
$$

with all other variables and parameters having same interpretation as in (2.1)-(2.3).

The analysis of the model (2.4) for the stability of its positive equilibria can provide an opportunity to see the effects of time delays in the production of Mdm2 and the degradation of P53 proteins on the nature of oscillations in the P53-Mdm2 network. But this analysis relates to locating the roots in the complex plane of a characteristic equation in the form of an exponential polynomial equation of the type $\sum_{i=0}^{m} f_{i}(\lambda) \exp \left(w_{i} \lambda\right)=0$, where $f_{i}(\lambda)$ is a polynomial in $\lambda$ and $w_{i}$ as $\tau_{1}, \tau_{2}, \tau_{3}$ or a combination of these such as $\tau_{1}+\tau_{2}, \tau_{1}+\tau_{3}, \tau_{2}+\tau_{3}, \tau_{1}+\tau_{2}+\tau_{3}$. This equation containing thirteen terms of the form $f_{i}(\lambda) \exp \left(w_{i} \lambda\right)$ turns out to be cumbersome to handle analytically. If we relax our assumption a bit and consider $\tau_{3}=0$ in model (2.4), the 
number of terms in the exponential polynomial equation $\sum_{i=0}^{m} f_{i}(\lambda) \exp \left(w_{i} \lambda\right)=0$ reduces from thirteen to four and its analysis becomes a bit simpler.The assumption $\tau_{3}=0$ is quite reasonable as the production of Mdm2 by P53 in the literature mostly has been considered direct without any intermediary proteins between them [9]. However, the degradation of P53 by Mdm2 follows different pathways that include the activation of various kinases such as ATM, Chk2, ATR, P14arf etc. which justifies considering delays $\tau_{1}$ and $\tau_{2}$ to be non zero. Furthermore, $\tau_{1}$ and $\tau_{2}$ may include the time required for the interaction between proteins P76MDM2 and P90MDM2 in addition to the time required for translocation of P53 in cytosol [24, 30, 31]. Thus introducing $\tau_{3}=0$ in model (2.4) for the purpose of simplicity but without comprising with the reality for the arguments cited as above, we shall consider the following model in this paper.

$$
\begin{aligned}
& \frac{d x}{d t}=a-s x-b x-c x z\left(t-\tau_{1}\right), \\
& \frac{d y}{d t}=s x-b y-d y z\left(t-\tau_{2}\right), \\
& \frac{d z}{d t}=h+f F(x+y)-g(s) z .
\end{aligned}
$$

The analysis of this model would certainly help compare P53-Mdm2 network stability and oscillations for delays in production of Mdm2 versus delays in degradation of P53.

Since model (2.5) represents dynamics of populations of plain P53, active P53 and Mdm2 proteins, it is natural and biologically feasible to assume that these populations remain positive and bounded. We discuss the positivity and boundedness of the solutions of the model (2.5) in the next section.

\section{Positivity and boundedness}

Let

$$
\max \left(\tau_{1}, \tau_{2}\right)=\tau
$$

Let $C$ denote the Banach space of continuous functions $\phi:[-\tau, 0] \rightarrow R^{3}$ with sup-norm

$$
\|\phi\|=\max \left\{\sup _{-\tau \leq \theta \leq 0}\left|\phi_{1}(\theta)\right|, \sup _{-\tau \leq \theta \leq 0}\left|\phi_{2}(\theta)\right|, \sup _{-\tau \leq \theta \leq 0}\left|\phi_{3}(\theta)\right|\right\},
$$

where

$$
\phi=\left(\phi_{1}, \phi_{2}, \phi_{3}\right)
$$

Let

$$
C_{+}=\left\{\phi=\left(\phi_{1}, \phi_{2}, \phi_{3}\right) \in C: \phi_{i} \geq 0, i=1,2,3 ; \theta \in[-\tau, 0]\right\} .
$$

The initial conditions for the model (2.5) are

$$
x(\theta)=\phi_{1}(\theta), y(\theta)=\phi_{2}(\theta), z(\theta)=\phi_{3}(\theta), \quad \theta \in[-\tau, 0] .
$$




\subsection{Positive invariance}

Lemma 3.1. Every solution of the model (2.5) with initial conditions (3.5) remains positive for all time $t>0$.

Proof. Denoting

$$
\begin{gathered}
W=(x, y, z)^{T} \in R_{+}^{3}, \\
G\left(W_{t}\right)=\left[\begin{array}{c}
G_{1}\left(W_{t}\right) \\
G_{2}\left(W_{t}\right) \\
G_{3}\left(W_{t}\right)
\end{array}\right]=\left[\begin{array}{c}
a-s x-b x-c x z\left(t-\tau_{1}\right) \\
s x-b y-d y z\left(t-\tau_{2}\right) \\
h+f F(x+y)-g(s) z
\end{array}\right],
\end{gathered}
$$

where the mapping $G: C_{+} \longrightarrow R_{+}^{3}$ is such that $G \in C^{\infty}\left(R_{+}^{3}\right)$, system (2.5) can be written in matrix form as

$$
\dot{W}=G\left(W_{t}\right)
$$

with $W_{t}(\theta)=W(t+\theta), \theta \in[-\tau, 0]$. For $W_{0}(\theta)=0, \theta \in[-\tau, 0], G_{1}=a \geq 0, G_{2}=0, G_{3}=$ $h \geq 0$; it follows from a lemma in Yang et al. [32] that any solution of (3.8) with $W_{0}(\theta) \in C_{+}$is such that $W(t) \in R_{+}^{3} \forall t \geq 0$.

\subsection{Boundedness}

The right hand side of the model system (2.5) is a Lipschitz continuous function. It thus implies that there exists a unique solution of model system (2.5) with initial conditions (3.5). Using the positivity of state variables we get from the first equation of the system (2.5)

$$
\frac{d x}{d t} \leq a-(s+b) x
$$

and it follows from the well known comparison principle that

$$
\sup _{t \rightarrow \infty} x(t) \leq \frac{a}{s+b} .
$$

Using first and second equations of (2.5) we get

$$
\frac{d(x+y)}{d t} \leq a-b(x+y)
$$

and hence

$$
\sup _{t \rightarrow \infty}(x+y) \leq \frac{a}{b}=M(\text { say }) .
$$

Now from the third equation of (2.5) it follows

$$
\frac{d z}{d t} \leq h+f F(M)-g(s) z,
$$


which yields

$$
\sup _{t \rightarrow \infty} z(t) \leq \frac{h+f F(M)}{g(s)} .
$$

It follows from (3.12) and (3.14) that solutions of model (2.5) with initial conditions (3.5) are bounded. Now Lemma (3.1) and the boundedness of solutions of model (2.5) yield the following result.

Theorem 3.1. Every solution of the model (2.5) with initial conditions (3.5) is positive and bounded.

\section{Results and discussion: stability analysis}

An equilibrium $E^{*}=\left(x^{*}, y^{*}, z^{*}\right)$ of the model (2.5) will be a solution of the following system of equations:

$$
\begin{aligned}
a-s x-b x-c x z & =0, \\
s x-b y-d y z & =0, \\
h+f F(x+y)-g z & =0 .
\end{aligned}
$$

Since the degradation function $g$ depends on $s$ and it will have different values depending on whether the system is stressed or not, we discuss the two cases in the following.

\subsection{Case 1: $\mathrm{s}=0$ (the system is not stressed)}

When $s=0$, the system (4.1) has a unique nonnegative equilibrium $E^{0}=\left(x^{0}, 0, z^{0}\right)$ where $x^{0}$ is a positive root of the 5 th degree equation:

$$
l x^{5}+m x^{4}+n x+p=0
$$

with

$$
\begin{aligned}
l & =\frac{g_{1} b}{c}+h+f>0, \\
m & =-\frac{a g_{1}}{c}<0, \\
n & =K^{4} \frac{g_{1} b}{c}+h>0, \\
p & =-\frac{K^{4} a g_{1}}{c}<0,
\end{aligned}
$$

and

$$
z^{0}=\frac{a-b x^{0}}{c x^{0}}
$$


such that $a-b x^{0}>0$.

Considering $x=x^{0}+\xi, y=\eta, z=z^{0}+\rho$ and linearizing the system (2.5) about $E^{0}$, we obtain

$$
\begin{aligned}
& \frac{d \xi}{d t}=-\left(b+c z^{0}\right) \xi-c x^{0} \rho\left(t-\tau_{1}\right), \\
& \frac{d \eta}{d t}=-\left(b+d z^{0}\right) \eta \\
& \frac{d \rho}{d t}=f F^{\prime}\left(x^{0}\right)(\xi+\eta)-g_{1} \rho,
\end{aligned}
$$

where $F^{\prime}$ denotes the derivative of $F$. The characteristic equation of this linearized system is given by

$$
\left(\lambda+d z^{0}+b\right)\left[\left(\lambda+g_{1}\right)\left(\lambda+c z^{0}+b\right)+c f x^{0} F^{\prime}\left(x^{0}\right) \exp \left(-\lambda \tau_{1}\right)\right]=0
$$

which has one eigenvalue $\lambda=-\left(d z_{0}+b\right)$ and the remaining eigenvalues will be roots of the transcendental equation

$$
\lambda^{2}+\left(g_{1}+c z^{0}+b\right) \lambda+g_{1}\left(c z^{0}+b\right)+c f x^{0} F^{\prime}\left(x^{0}\right) \exp \left(-\lambda \tau_{1}\right)=0
$$

The characteristic equation (4.10) is same as characteristic equation (5) in [25]. The authors in [25] comment that this equation is a transcendental equation which can only be studied numerically. We study this equation analytically based on the approach in [33]. This analysis provides the following stability bifurcation result for equilibrium $E^{0}$.

Theorem 4.1. (i) If $g_{1} \geq \frac{c}{a}\left(x^{0}\right)^{2} f F^{\prime}\left(x^{0}\right)$, then there are no stability switches and $E^{0}$ is asymptotically stable for $\tau_{1}>0$.

(ii) If $g_{1}<\frac{c}{a}\left(x^{0}\right)^{2} f F^{\prime}\left(x^{0}\right)$, then $E^{0}$ is uniformly asymptotically stable for $\tau_{1}<\tau_{1,0}$ and unstable for $\tau_{1}>\tau_{1,0}$, such that

$$
\tau_{1,0}=\frac{\theta}{w_{+}}
$$

where $\theta$ and $w_{+}$satisfy

$$
\begin{aligned}
w_{+}^{2} & =\frac{\left.-\left(g_{1}^{2}+\left(c z^{0}+b\right)^{2}\right)+\sqrt{\left(g_{1}^{2}-\left(c z^{0}+b\right)^{2}\right)^{2}+4\left(c f x^{0} F^{\prime}\left(x^{0}\right)\right.}\right)^{2}}{2}, \\
\cos \theta & =\frac{w_{+}^{2}-g_{1}\left(c z^{0}+b\right)}{c f x^{0} F^{\prime}\left(x^{0}\right)} .
\end{aligned}
$$

(This result provides thresholds on degradation rate $g=g_{1}$ of $M d m 2$ and the delay $\tau_{1}$ for stability or switch in stability of the equilibrium $E^{0}$. More specifically, it implies that for large degradation rates $g_{1}$ of $M d m 2$ the system always tends to stability but lower values of $g_{1}$ may produce oscillations in plain P53 and Mdm2 proteins.) 
Proof. Obviously, $\lambda=0$ is not a root of equation (4.10) since $g_{1}\left(c z^{0}+b\right)+c f x^{0} F^{\prime}\left(x^{0}\right) \neq 0$. Furthermore, for $\tau_{1}=0$, all the roots $\lambda$ of the quadratic equation (4.10) are such that $\operatorname{Re}(\lambda)<0$. Suppose $\lambda=i w, w>0$ is a root of (4.10), then substituting it in (4.10) it becomes

$$
(i w)^{2}+\left(g_{1}+c z^{0}+b\right)(i w)+g_{1}\left(c z^{0}+b\right)+c f x^{0} F^{\prime}\left(x^{0}\right) \exp \left(-i w \tau_{1}\right)=0 .
$$

Separating real and imaginary parts of (4.14) we have

$$
\begin{aligned}
g_{1}\left(c z^{0}+b\right)-w^{2}+c f x^{0} F^{\prime}\left(x^{0}\right) \cos \left(w \tau_{1}\right) & =0, \\
\left(g_{1}+c z^{0}+b\right) w-c f x^{0} F^{\prime}\left(x^{0}\right) \sin \left(w \tau_{1}\right) & =0 .
\end{aligned}
$$

Squaring and adding equations (4.15) and (4.16), we get a fourth degree equation in $w$ as follows

$$
w^{4}+L w^{2}+M_{1}=0,
$$

where

$$
L=g_{1}^{2}+\left(c z^{0}+b\right)^{2}>0
$$

and

$$
M_{1}=g_{1}^{2}\left(c z^{0}+b\right)^{2}-\left(c f x^{0} F^{\prime}\left(x^{0}\right)\right)^{2} .
$$

The roots of (4.17) are given by

$$
w_{ \pm}^{2}=\frac{-L \pm \sqrt{L^{2}-4 M_{1}}}{2} .
$$

Since $L>0$, it can be seen that equation (4.17) has no real root $w$ such that $w>0$ when $M_{1} \geq 0$. Thus roots of equation (4.10) would not cross over the imaginary axis and lie only in the left half plane of the complex plane when $M_{1} \geq 0\left(g_{1} \geq \frac{c}{a}\left(x^{0}\right)^{2} f F^{\prime}\left(x^{0}\right)\right)$ for $\tau_{1}>0$. Hence part $(i)$ of the theorem is established.

If $M_{1}<0$ (or $g_{1}<\frac{c}{a}\left(x^{0}\right)^{2} f F^{\prime}\left(x^{0}\right)$ ), the only real roots of (4.20) that would satisfy (4.17) are $w_{+}^{2}=\frac{-L+\sqrt{\left(L^{2}-4 M_{1}\right)}}{2}$. Thus there exists a unique positive real $w=w_{+}$of (4.17) and hence implying one corresponding pure imaginary root $\lambda=i w_{+}$of equation (4.10). Substituting $w=w_{+}$in equation (4.15), we get

$$
\cos \left(w_{+} \tau_{1}\right)=\frac{w_{+}^{2}-g_{1}\left(c z^{0}+b\right)}{c f x^{0} F^{\prime}\left(x^{0}\right)}=\cos \theta(\text { say }) .
$$

It thus implies

$$
\tau_{1, n}=\left(\frac{1}{w_{+}}\right)(\theta+2 n \pi), n=0,1,2, \cdots
$$

The minimum of $\tau_{1}$ can be obtained by putting $n=0$ in equation (4.22). At $\tau_{1,0}$, the root $\lambda=i w_{+}$ will cross the imaginary axis the first time and thus $\tau_{1,0}$ will be the threshold for bifurcation in the stability of $E^{0}$. This establishes part $(i i)$ of the theorem. This also completes the proof of the Theorem (4.1). 


\subsection{Case 2: $s \neq 0$ (the system is stressed)}

When $s \neq 0$, the solution of the system (4.1) gives the equilibrium $E^{*}=\left(x^{*}, y^{*}, z^{*}\right)$, where

$$
\begin{aligned}
& x^{*}=\frac{a}{s+b+c z^{*}}, \\
& y^{*}=\frac{s x^{*}}{b+d z^{*}},
\end{aligned}
$$

and $z^{*}$ is the unique positive root of the ninth degree equation in $z$

$$
\frac{1}{K^{4}}\left(\frac{f}{g z-h}-1\right)=\left[\frac{(s+b+c z)(b+d z)}{a(s+b+d z)}\right]^{4} .
$$

Considering the transformations $x=x^{*}+\xi, y=y^{*}+\eta$ and $z=z^{*}+\rho$, the linearized system of (2.5) about $E^{*}$ is obtained as

$$
\begin{aligned}
& \frac{d \xi}{d t}=-\left(s+b+c z^{*}\right) \xi-c x^{*} \rho\left(t-\tau_{1}\right), \\
& \frac{d \eta}{d t}=s \xi-\left(b+d z^{*}\right) \eta-d y^{*} \rho\left(t-\tau_{2}\right), \\
& \frac{d \rho}{d t}=f F^{\prime}\left(x^{*}+y^{*}\right)(\xi+\eta)-g \rho .
\end{aligned}
$$

The characteristic equation for this linearized system is

$$
\lambda^{3}+a_{1} \lambda^{2}+a_{2} \lambda+a_{3}+\left(b_{1} \lambda+b_{2}\right) \exp \left(-\tau_{2} \lambda\right)+\left(c_{1} \lambda+c_{2}\right) \exp \left(-\tau_{1} \lambda\right)=0
$$

where

$$
\begin{aligned}
& a_{1}=g+2 b+s+(c+d) z^{*}, \\
& a_{2}=g\left(b+d z^{*}\right)+\left(s+b+c z^{*}\right)\left(g+b+d z^{*}\right), \\
& a_{3}=g\left(s+b+c z^{*}\right)\left(b+d z^{*}\right), \\
& b_{1}=d y^{*} f F^{\prime}\left(x^{*}+y^{*}\right), \\
& b_{2}=d y^{*} f F^{\prime}\left(x^{*}+y^{*}\right)\left(s+b+c z^{*}\right), \\
& c_{1}=c x^{*} f F^{\prime}\left(x^{*}+y^{*}\right), \\
& c_{2}=c x^{*} f F^{\prime}\left(x^{*}+y^{*}\right)\left(s+b+d z^{*}\right) .
\end{aligned}
$$

In (4.28), all the parameters $a_{i}, b_{i}$ and $c_{i}$ are positive.

Remark 1. Equation (4.27) is new in that it is a transcendental equation, cubic in $\lambda$ in non-delay terms and it has two delays. To the best of our knowledge, this equation has not been studied in the literature. 
We study the stability of $E^{*}$ by using Nyquist criterion following Freedman and Rao [34] and obtain the following result:

Theorem 4.2. Consider (4.28) and let $\tau=\max \left(\tau_{1}, \tau_{2}\right)$. The positive equilibrium $E^{*}$ is locally asymptotically stable under any of the following conditions

(i) $a_{2}>v_{+}^{2}+b_{1}+c_{1}$ and $\tau<\tau_{1}^{*}$,

(ii) $v_{+}^{2}-b_{1}-c_{1}<a_{2}<v_{+}^{2}+b_{1}+c_{1}$ and $\tau<\tau_{2}^{*}$,

where,

$$
\begin{aligned}
v_{+} & =\frac{\left(b_{1}+c_{1}\right)+\sqrt{\left(b_{1}+c_{1}\right)^{2}+4 a_{1}\left(a_{3}+b_{2}+c_{2}\right)}}{2 a_{1}}, \\
\tau_{1}^{*} & =\frac{-v_{+}^{2}+a_{2}-b_{1}-c_{1}}{b_{2}+c_{2}} \\
\tau_{2}^{*} & =\frac{\eta}{b_{2}+c_{2}}
\end{aligned}
$$

and $\eta$ is such that

$$
0<\eta<-v_{+}^{2}+a_{2}+b_{1}+c_{1} \text { and } \cos \tau v_{+}=\frac{\eta+v_{+}^{2}-a_{2}}{b_{1}+c_{1}}
$$

(Result (i) provides upper bound $\tau_{1}^{*}$ on $\tau=\max \left(\tau_{1}, \tau_{2}\right)$ such that any delays $\tau_{1}, \tau_{2}$ in model (2.5) with $\tau_{1}, \tau_{2}$ less than or equal to $\tau_{1}^{*}$ ensure stability of its equilibrium $E^{*}$. This suggests that any oscillatory behavior in plain P53, active P53 and Mdm2 can be expected only when either $\tau_{1}$ or $\tau_{2}$ or both $\tau_{1}$ and $\tau_{2}$ are greater than $\tau_{1}^{*}$. Result (ii) provides another upper bound $\tau_{2}^{*}$ on $\tau=\max \left(\tau_{1}, \tau_{2}\right)$ under different condition on model parameters.)

Proof. It can be noted that $\lambda=0$ is not a root of (4.27) since $a_{3}+b_{2}+c_{2} \neq 0$. If $\lambda=u+i v$ satisfies (4.27), then $u$ and $v$ are real solutions of

$$
\begin{array}{r}
u^{3}-3 u v^{2}+a_{1}\left(u^{2}-v^{2}\right)+a_{2} u+a_{3}+\left(\left(c_{1} u+c_{2}\right) \cos \left(v \tau_{1}\right)+c_{1} v \sin \left(v \tau_{1}\right)\right) \exp \left(-\tau_{1} u\right)+ \\
\left(\left(b_{1} u+b_{2}\right) \cos \left(v \tau_{2}\right)+b_{1} v \sin \left(v \tau_{2}\right)\right) \exp \left(-\tau_{2} u\right)=0,
\end{array}
$$

and

$$
\begin{aligned}
& 3 u^{2} v-v^{3}+2 a_{1} u v+a_{2} v+\left(c_{1} v \cos \left(v \tau_{1}\right)-\left(c_{1} u+c_{2}\right) \sin \left(v \tau_{1}\right)\right) \exp \left(-\tau_{1} u\right) \\
&+\left(b_{1} v \cos \left(v \tau_{2}\right)-\left(b_{1} u+b_{2}\right) \sin \left(v \tau_{2}\right)\right) \exp \left(-\tau_{2} u\right)=0 .
\end{aligned}
$$


We note that in the absence of delays that is when $\tau_{1}=0$ and $\tau_{2}=0$, the characteristic equation (4.27) reduces to

$$
\lambda^{3}+a_{1} \lambda^{2}+\left(a_{2}+b_{1}+c_{1}\right) \lambda+a_{3}+b_{2}+c_{2}=0 .
$$

Using Routh-Hurwitz criteria, it can be shown that all roots $\lambda$ of the equation (4.35) are such that $\operatorname{Re}(\lambda)<0$. Furthermore, since $\lambda=0$ is not a root of (4.27), it follows that the equilibrium $E^{*}$ of our model (2.5) is always asymptotically stable in the absence of delays. By continuity, all eigenvalues of (4.27) will have negative real parts for sufficiently small $\tau_{i}>0(i=1,2)$ provided that no eigenvalues bifurcate with real parts from $+\infty$. We shall assume that this condition holds in our case. Following analysis of [34] for the Nyquist criterion, range of delay can be estimated for which $E^{*}$ will remain locally asymptotically stable.

Let us consider system (4.26) and the space of all real valued continuous functions (defined on $\left.[-\tau, \infty), \tau=\max \left(\tau_{1}, \tau_{2}\right)\right)$ satisfying the initial conditions $\xi(t)=0, \eta(t)=0, \rho(t)=0$ for $t<0, \xi\left(0^{+}\right)=k_{1} \neq 0, \eta\left(0^{+}\right)=k_{2} \neq 0, \rho\left(0^{+}\right)=k_{3} \neq 0$. Let $U(S), V(S)$, and $W(S)$ denote the Laplace transforms of $\xi(t), \eta(t)$, and $\rho(t)$ respectively. Then taking the Laplace transform of (4.26), we get

$$
\begin{aligned}
& S U(S)=-\left(s+b+c z^{*}\right) U(S)-c x^{*} \exp \left(-\tau_{1} S\right) W(S)+k_{1}, \\
& S V(S)=s U(S)-\left(b+d z^{*}\right) V(S)-d y^{*} \exp \left(-\tau_{2} S\right) W(S)+k_{2}, \\
& S W(S)=f F^{\prime}\left(x^{*}+y^{*}\right)(U(S)+V(S))-g W(S)+k_{3} .
\end{aligned}
$$

Eliminating $V$ and $W$ from (4.36) and using (4.28), we obtain

$$
\begin{aligned}
U(S)\left[S^{3}+a_{1} S^{2}+\right. & \left.a_{2} S+a_{3}+\left(b_{1} S+b_{2}\right) \exp \left(-\tau_{2} S\right)+\left(c_{1} S+c_{2}\right) \exp \left(-\tau_{1} S\right)\right] \\
& =-k_{3}\left[c x^{*}\left(S+b+d z^{*}\right)\right] \exp \left(-\tau_{1} S\right)-k_{2} c x^{*} f F^{\prime} \exp \left(-\tau_{1} S\right) \\
& +k_{1} f F^{\prime} \exp \left(-\tau_{2} S\right)+k_{1}\left[S^{2}+S\left(g+b+d z^{*}\right)+g\left(b+d z^{*}\right)\right] .
\end{aligned}
$$

If $U(S)$ has poles with positive real parts, then the inverse Laplace transform of $U(S)$ will have terms which exponentially increase with time. In order for $E^{*}$ to be locally asymptotically stable, it is necessary and sufficient that all poles of $U(S)$ have negative real parts. Using the Nyquistplot technique (see $[21,34]$ for details), it can be seen that the conditions for the local asymptotic stability of $E^{*}$ are given as

$$
\begin{aligned}
& \text { (i) } \operatorname{ImH}\left(i v_{0}\right)>0 \\
& (i i) \quad \operatorname{ReH}\left(i v_{0}\right)=0
\end{aligned}
$$

where

$$
H(S)=S^{3}+a_{1} S^{2}+a_{2} S+a_{3}+\left(b_{1} S+b_{2}\right) \exp \left(-\tau_{2} S\right)+\left(c_{1} S+c_{2}\right) \exp \left(-\tau_{1} S\right),
$$


and $v_{0}$ is the smallest positive root of the equation $(4.38(i i))$.

The Nyquist criteria in our case reduces to the following statement:

If $v_{0}$ is a solution of the equation (4.33) with $u=0$, i.e., $v_{0}$ satisfies

$$
-a_{1} v_{0}^{2}+a_{3}+b_{2} \cos \left(v_{0} \tau_{2}\right)+b_{1} v_{0} \sin \left(v_{0} \tau_{2}\right)+c_{2} \cos \left(v_{0} \tau_{1}\right)+c_{1} v_{0} \sin \left(v_{0} \tau_{1}\right)=0
$$

then the inequality

$$
\phi\left(\tau_{1}, \tau_{2}, v_{0}\right)>\psi\left(\tau_{1}, \tau_{2}, v_{0}\right)
$$

with

$$
\phi\left(\tau_{1}, \tau_{2}, v_{0}\right)=-v_{0}^{3}+a_{2} v_{0}+b_{1} v_{0} \cos \left(v_{0} \tau_{2}\right)+c_{1} v_{0} \cos \left(v_{0} \tau_{1}\right)
$$

and

$$
\psi\left(\tau_{1}, \tau_{2}, v_{0}\right)=b_{2} \sin \left(v_{0} \tau_{2}\right)+c_{2} \sin \left(v_{0} \tau_{1}\right)
$$

must hold.

By symmetry it follows that if $v_{0}$ satisfies (4.40) then so does $-v_{0}$. Hence without loss of generality $v_{0}$ can be assumed such that $v_{0}>0$. Noting from (4.40) the following

$$
\begin{aligned}
a_{1} v_{0}^{2} & =a_{3}+b_{2} \cos \left(v_{0} \tau_{2}\right)+b_{1} v_{0} \sin \left(v_{0} \tau_{2}\right)+c_{2} \cos \left(v_{0} \tau_{1}\right)+c_{1} v_{0} \sin \left(v_{0} \tau_{1}\right) \\
& \leq a_{3}+b_{2}+b_{1} v_{0}+c_{2}+c_{1} v_{0}
\end{aligned}
$$

and denoting $v_{+}$the positive root of $a_{1} v^{2}-\left(b_{1}+c_{1}\right) v-\left(a_{3}+b_{2}+c_{2}\right)=0$, such that

$$
v_{+}=\frac{\left(b_{1}+c_{1}\right)+\sqrt{\left(b_{1}+c_{1}\right)^{2}+4 a_{1}\left(a_{3}+b_{2}+c_{2}\right)}}{2 a_{1}},
$$

it follows that $v_{0} \leq v_{+}$. Thus $v_{+}$is the upper bound of $v_{0}$.

Now if $\tilde{\phi}\left(\tau_{1}, \tau_{2}\right)$ and $\tilde{\psi}\left(\tau_{1}, \tau_{2}\right)$ can be found such that

$$
\frac{\phi\left(\tau_{1}, \tau_{2}, v_{0}\right)}{\tau v_{0}} \geq \tilde{\phi}\left(\tau_{1}, \tau_{2}\right)>\tilde{\psi}\left(\tau_{1}, \tau_{2}\right) \geq \frac{\psi\left(\tau_{1}, \tau_{2}, v_{0}\right)}{\tau v_{0}}
$$

for $\tau<\tau^{*}$ and $0<v_{0} \leq v_{+}$, then Nyquist criteria (i.e., (4.40) and (4.41)) holds for those values of $\tau_{1}$ and $\tau_{2}$. The upper bound $\tau^{*}$ for delay $\tau$ is estimated in the following:

From (4.43),

$$
\frac{\psi\left(\tau_{1}, \tau_{2}, v_{0}\right)}{\tau v_{0}} \leq b_{2}+c_{2}
$$

hence we choose $\tilde{\psi}\left(\tau_{1}, \tau_{2}\right)=b_{2}+c_{2}$.

From (4.42),

$$
\frac{\phi\left(\tau_{1}, \tau_{2}, v_{0}\right)}{\tau v_{0}}=\frac{-v_{0}^{2}+a_{2}+b_{1} \cos \left(v_{0} \tau_{2}\right)+c_{1} \cos \left(v_{0} \tau_{1}\right)}{\tau}
$$




$$
\begin{aligned}
& \geq \frac{-v_{+}^{2}+a_{2}+b_{1} \cos \left(v_{0} \tau_{2}\right)+c_{1} \cos \left(v_{0} \tau_{1}\right)}{\tau} \\
& \geq \frac{-v_{+}^{2}+a_{2}-b_{1}-c_{1}}{\tau}=\tilde{\phi}\left(\tau_{1}, \tau_{2}\right)(\text { say }) .
\end{aligned}
$$

Supposing $a_{2}>v_{+}^{2}+b_{1}+c_{1}$ and $\tau<\frac{-v_{+}^{2}+a_{2}-b_{1}-c_{1}}{b_{2}+c_{2}}$, it follows from (4.48) that

$$
\frac{\phi\left(\tau_{1}, \tau_{2}, v_{0}\right)}{\tau v_{0}} \geq \tilde{\phi}\left(\tau_{1}, \tau_{2}\right)>b_{2}+c_{2}=\tilde{\psi}\left(\tau_{1}, \tau_{2}\right) \text {. }
$$

Thus the Nyquist criterion is satisfied when $a_{2}>v_{+}^{2}+b_{1}+c_{1}$ and $\tau<\frac{-v_{+}^{2}+a_{2}-b_{1}-c_{1}}{b_{2}+c_{2}}$. This establishes part (i) of the theorem.

Next we consider the case when $a_{2} \leq v_{+}^{2}+b_{1}+c_{1}$. Let us choose $\eta>0$ and $a_{2}$ such that

$$
\text { (i) } v_{+}^{2}-b_{1}-c_{1}<a_{2}<v_{+}^{2}+b_{1}+c_{1}, \quad(i i)-v_{+}^{2}+a_{2}+b_{1}+c_{1}-\eta>0,
$$

and let

$$
\cos \tau v_{+}=\frac{\eta+v_{+}^{2}-a_{2}}{b_{1}+c_{1}}
$$

then using (4.48), it follows that

$$
\begin{aligned}
\frac{\phi\left(\tau_{1}, \tau_{2}, v_{0}\right)}{\tau v_{0}} & \geq \frac{-v_{+}^{2}+a_{2}+b_{1} \cos \left(v_{0} \tau_{2}\right)+c_{1} \cos \left(v_{0} \tau_{1}\right)}{\tau} \\
& \geq \frac{-v_{+}^{2}+a_{2}+b_{1} \cos \left(\tau v_{+}\right)+c_{1} \cos \left(\tau v_{+}\right)}{\tau} \\
& =\frac{\eta}{\tau}=\tilde{\phi}\left(\tau_{1}, \tau_{2}\right)(\text { say }),
\end{aligned}
$$

after invoking (4.50) and (4.51). Now if $\tau<\frac{\eta}{b_{2}+c_{2}}$ then the Nyquist criterion is satisfied. This establishes part (ii) of the theorem and this completes the proof of the Theorem (4.2).

\section{Numerical simulations}

With a purpose to justify the stability results of this paper and to compare them with the results of Bottani and Grammaticos model (2.1), we present in the following some numerical simulations. All simulations are done using MATLAB with the fixed set of parameter values: $a=1, b=$ $0.0001, c=0.001, d=0.0001, h=0$ or $0.1, f=1$, and $K=28$, same as considered in [25]. $\mathrm{Mdm} 2$ degradation in the absence of stress with $s=0$ is considered to be $g_{1}=0.00016$. In the presence of stress mainly due to DNA damage with $s=1$, it is taken to be $g_{2}=10 g_{1}$.

Graphs of Figure 1 and Figure 2 correspond to the results of Theorem 4.1 when the system is not stressed $(s=0)$. Figure 3 and Figure 4 represent results of Theorem 4.2 when the system is 
stressed $(s=1)$. In all Figures, Bottani and Grammaticos model (2.1) has been abbreviated as BG model. $h=0$ in these Figures means Mdm2 has no basal growth and $h \neq 0$ (taken here $h=0.1$ ) represents it has basal growth. Figure 1 contains comparative graphs corresponding to result of Theorem 4.1 (i) for densities of plain P53 and Mdm2 our model (2.5) versus BG model (2.1) for $s=0, h=0.1, g_{1}=0.00016$, and values of other parameters fixed as given above. The equilibrium $E^{0}$ for each model is $\left(x^{0}, y^{0}, z^{0}\right)=(1.5996,0,625.0666)$. Since $a g_{1}-c\left(x^{0}\right)^{2} f F^{\prime}\left(x^{0}\right) \geq 0$, it implies that $E^{0}$ is locally asymptotically stable for all $\tau_{1}>0$. Figure 1 shows that plain P53 and Mdm2 densities approach to $E^{0}$ for $\tau_{1}=4800$, a value of delay considered in BG model. Figure 2 has been drawn with parameter values same as in Figure 1 except that $h=0$ and its graphs correspond to the result of Theorem 4.1(ii) with $\tau_{1,0}=2159.7$. Figure 2(a) shows approach of densities of plain P53 and Mdm2 to $E^{0}$ when $\tau_{1}=2158<\tau_{1,0}$. Graphs of Figure 2(b) correspond to an unstable case when $\tau_{1}=4800>\tau_{1,0}$.

Figure 3 (a) has been plotted for $s=1, h=0.1$ and $g=g_{2}=0.0016$ keeping all other parameters fixed as earlier. The graphs of this figure correspond to the result of Theorem 4.2 (i). For this set of parameter values the positive equilibrium $E^{*}=(0.7628,24.4661,310.7900)$ and threshold $\tau_{1}^{*}=281.860$. Graphs of this figure show densities of plain P53, active P53 and Mdm2 approaching to $E^{*}$ when $\tau_{1}=\tau_{2}=280<\tau<\tau_{1}^{*}$. Graphs of Figure 3(b) represent the unstable case when $\tau_{1}=\tau_{2}=4800>\tau_{1}^{*}$, a delay considered in BG model (2.1) for comparison purpose. Figure 4 also corresponds to the result of Theorem 4.2 (i) with same set of parameter values as in Figure 3 except for $h=0$ i.e. without basal growth of Mdm2. The equilibrium $E^{*}$ in this case is $(0.7735,26.347,292.601)$ with threshold $\tau_{1}^{*}=257.786$. Figure 4 (a) corresponds to the case when $\tau_{1}=\tau_{2}=256<\tau_{1}^{*}$ in that all the proteins plain P53, active P53 and Mdm2 approach $E^{*}$. Figure 4 (b) corresponds to the case when $E^{*}$ is unstable with $\tau_{1}=\tau_{2}=4800>\tau_{1}^{*}$.

From these simulations, following observations may be highlighted:

- Time delays in the reproduction process in Mdm2 considered in earlier studies and the time delays in the degradation process in plain P53 and active P53 considered in this study although seem to retain basic nature of dynamics of P53-Mdm2 loop (i.e proteins P53 and Mdm2 either approach to stable state or display oscillatory behavior) but with a big difference in the early stages of the dynamics both in absence and presence of stress. This difference in early stages in our model is in terms of slow oscillatory behavior with much lower peaks than in BG model with the oscillatory dynamics catching up with BG model in later stages. This difference can be noted in all figures except Figure 1 when the system is not stressed $(s=0), \mathrm{Mdm} 2$ has basal growth and the degradation rate $\left(g_{1}\right)$ of $\mathrm{Mdm} 2$ is greater than a definite threshold value. 
- Comparing Figures 3 and 4 for large times, it can be noted that our model and BG model has almost similar dynamics both in stability and oscillatory behavior but with a difference in the behavior of active P53. It attains higher peaks when Mdm2 has no basal growth $(h=0)$ than when Mdm2 has basal growth $(h=0.1)$. Under stressed condition with DNA damage (Figures 3 and 4), it can be noted that switch in the nature of dynamics from stability to oscillations is delayed if $\mathrm{Mdm} 2$ has basal growth.

- Mdm2 dynamics in early stages in BG model and in our study under stressed condition of DNA damage seems to be evolving in opposite direction with peaks of oscillations in our study being lower than BG model irrespective of whether Mdm2 has basal growth or not (Figures 3 and 4). Furthermore, the oscillatory dynamics of Mdm2 in our study and BG model seems to be evolving in different phases. 

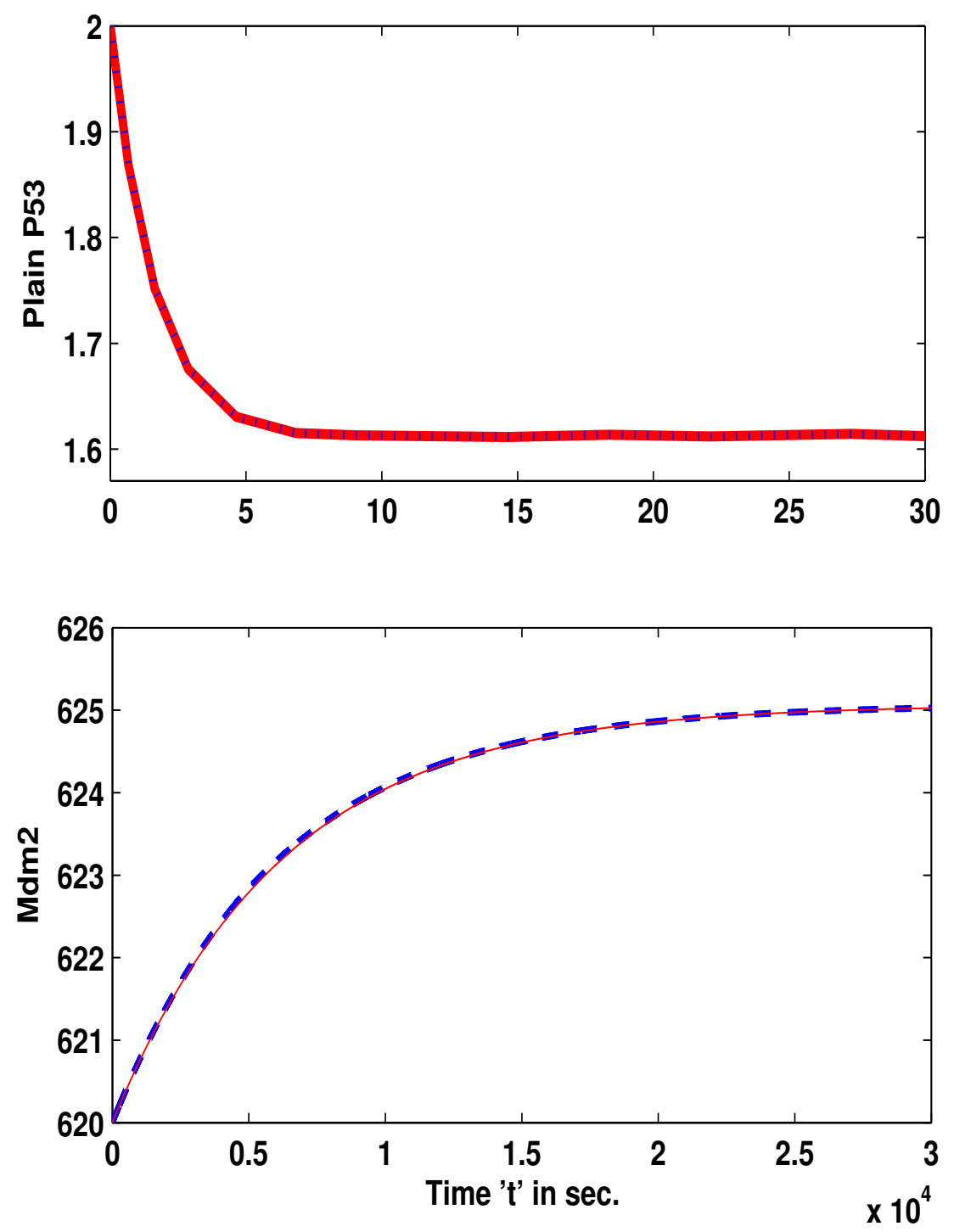

Figure 1: Comparative plots for plain P53 and Mdm2 our model (2.5) (red continuous line) versus BG model (2.1) (blue broken line). These graphs correspond to the results of Theorem 4.1(i). Section 5 has details of parameter values used in this figure with $s=0, h=0.1, \tau_{1}=4800$. 
(a)
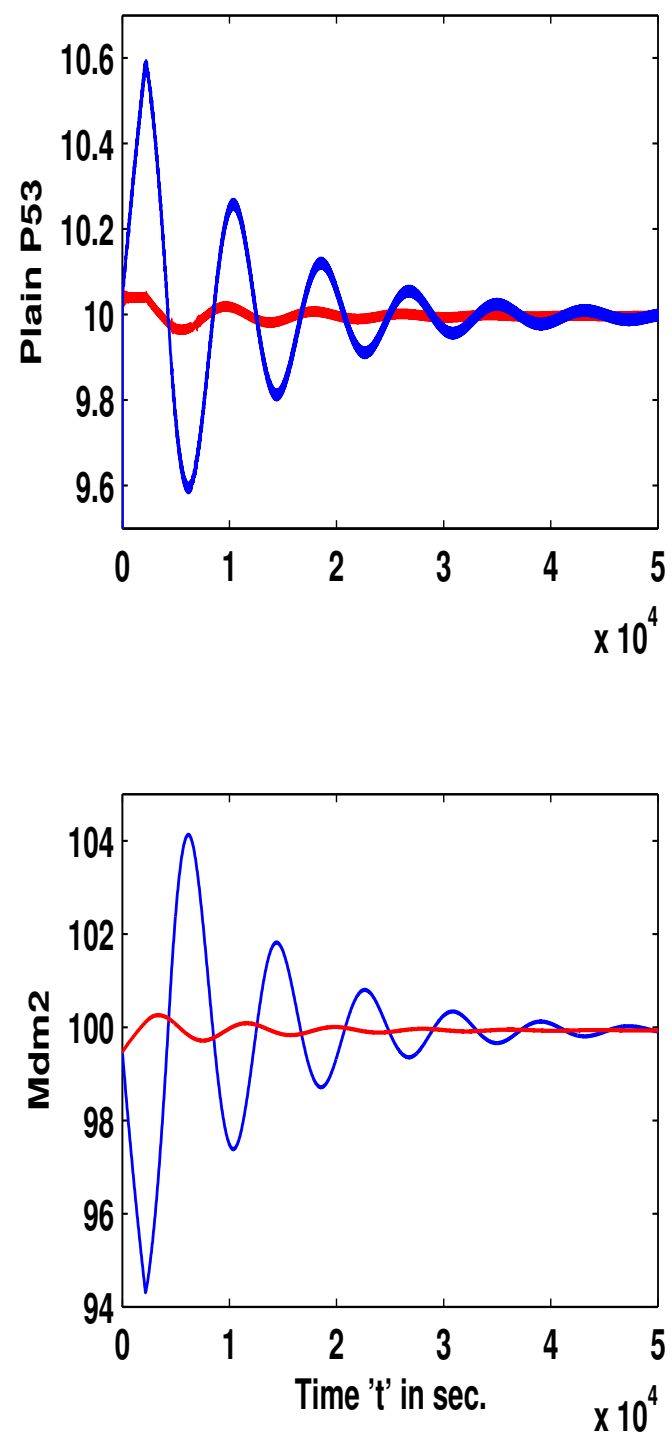

(b)

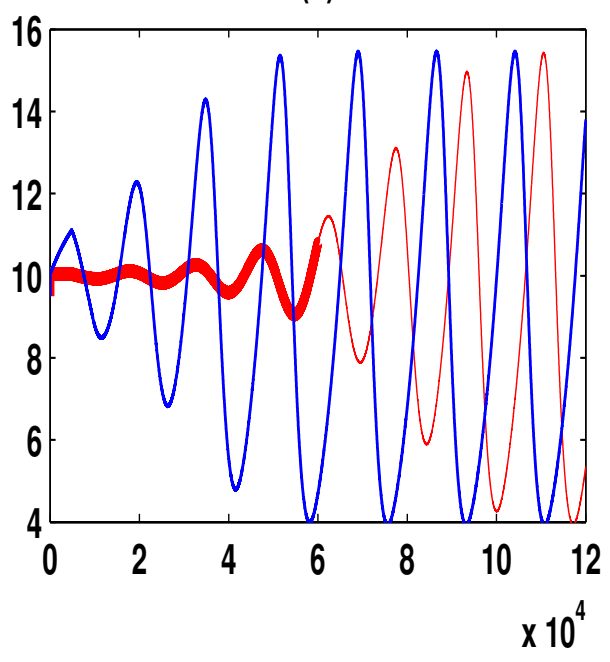

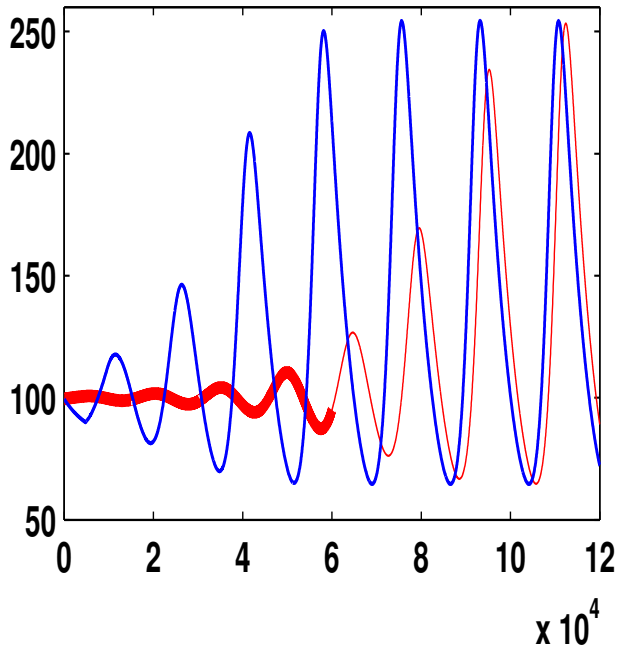

Figure 2: Comparative plots for plain P53 and Mdm2 our model (2.5) (red continuous line) versus BG model (2.1) (blue broken line). These graphs correspond to the results of Theorem 4.1(ii). Section 5 has details of parameter values used in this figure with (a) plotted for $s=0, h=0, \tau_{1}=2158$ and (b) for $s=0, h=0, \tau_{1}=4800$. 
(a)
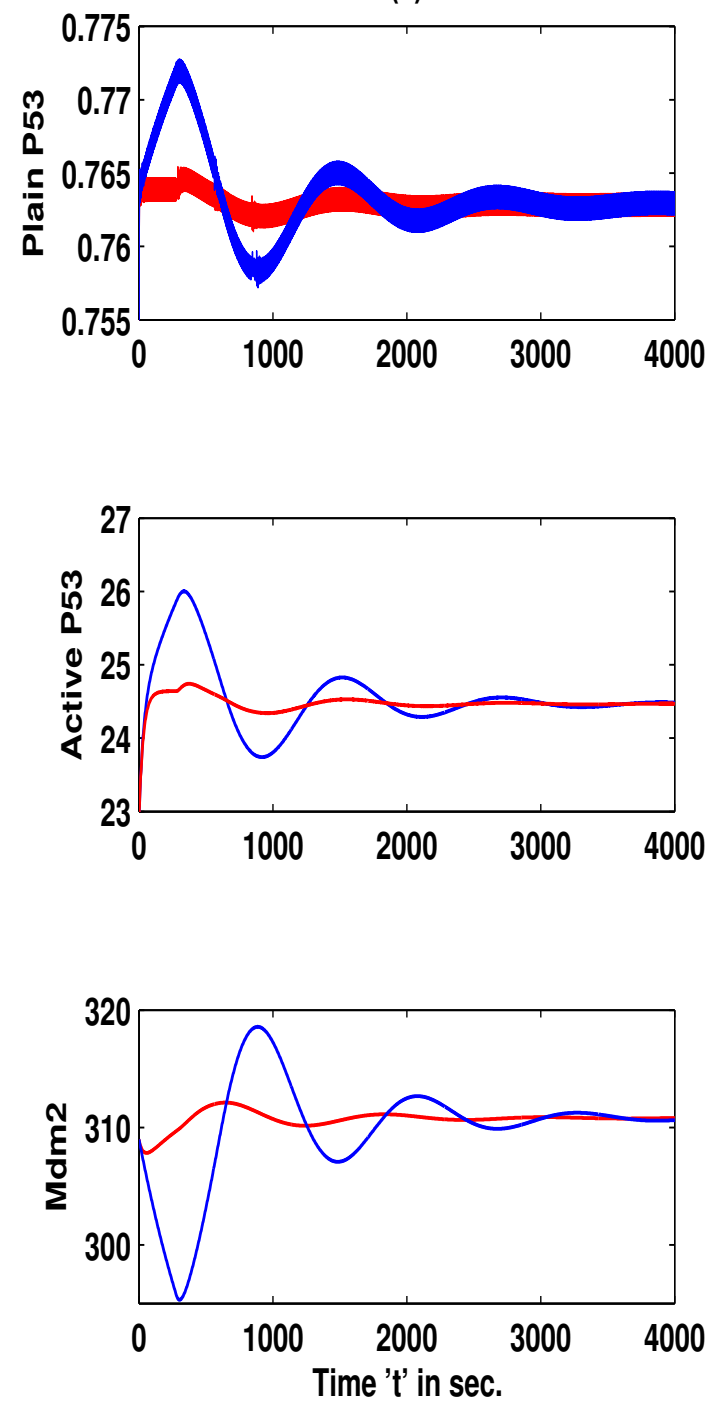

(b)
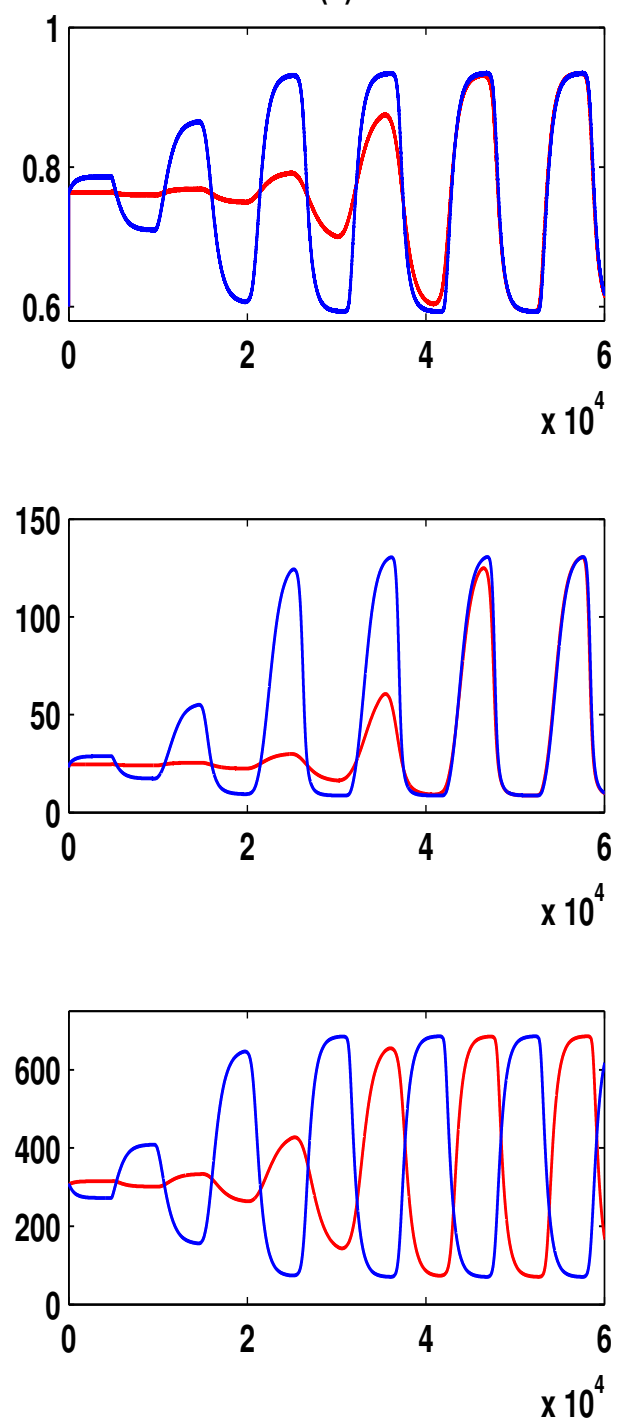

Figure 3: Comparative plots for plain P53, active P53 and Mdm2 our model (2.5) (red continuous line) versus BG model (2.1) (blue broken line). These graphs correspond to the results of Theorem 4.2(i). Parameter values used in this figure are given in section 5 with (a) plotted for $s=1, h=0.1, \tau_{1}=280, \tau_{2}=280$ and (b) for $s=1, h=0.1, \tau_{1}=4800, \tau_{2}=4800$. 
(a)
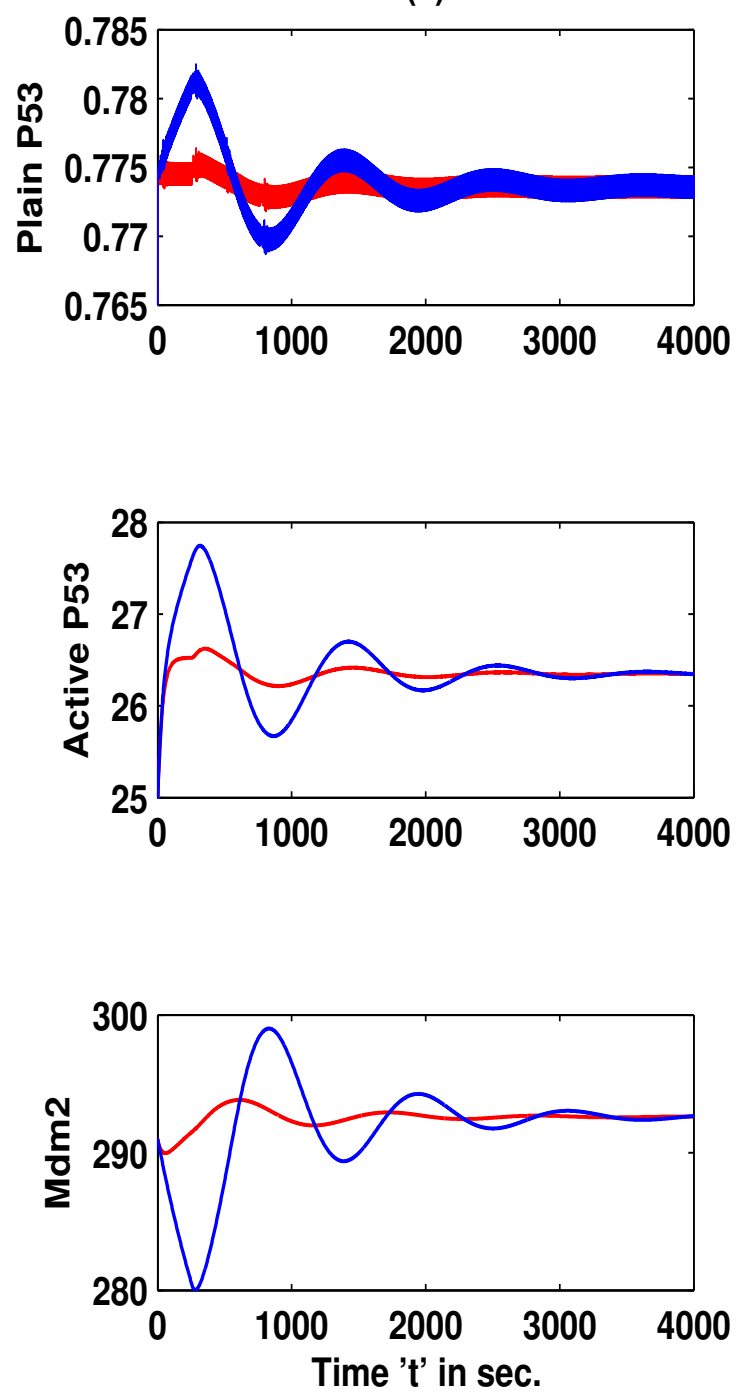

(b)
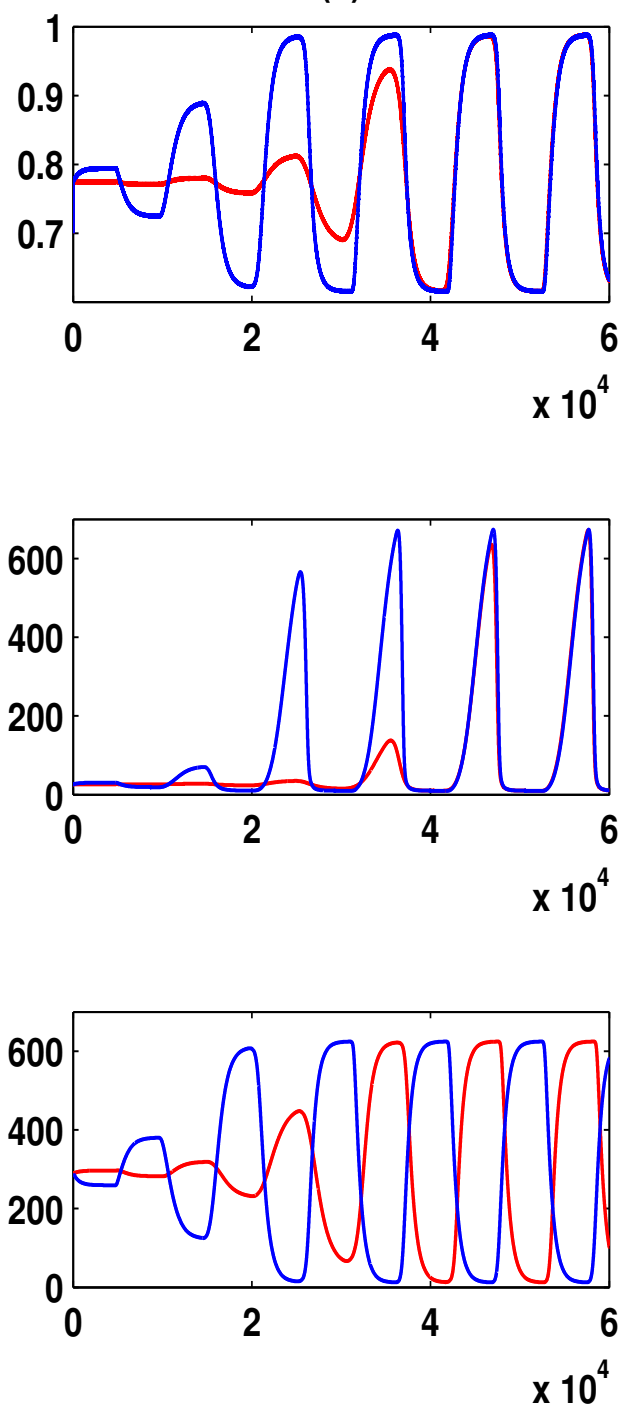

Figure 4: Comparative plots for plain P53, active P53 and Mdm2 our model (2.5) (red continuous line) versus BG model (2.1) (blue broken line). These graphs correspond to the results of Theorem 4.2 (i). Parameter values used in this figure are given in section 5 with (a) plotted for $s=1, h=0, \tau_{1}=256, \tau_{2}=256$ and (b) for $s=1, h=0, \tau_{1}=4800, \tau_{2}=4800$. 


\section{Conclusion}

In this paper, the stability behavior of P53-Mdm2 negative feedback loop is studied under two situations: (i) when the system is stressed (represented by binary parameter $s=1$ ) and (ii) when it is not $(s=0)$. The system is supposed to be in stressed condition due to DNA damage. In most of earlier studies, P53 induced transcriptional delay in the production of Mdm2 has been considered. This paper analyses the Mdm2 induced delay effect in the degradation of P53 and presents its comparative study with Bottani and Grammaticos model (2.1) in both cases $s=0$ and $s=1$. When $s=0$, it is observed that the natural degradation rate $g_{1}$ of Mdm2 plays an important role in the dynamical behavior of the system. It is found that for values of $g_{1}$ greater than a definite threshold the model system is stable and all its solutions tend to the equilibrium $E^{0}$. However, lower values of $g_{1}$ may produce oscillations in plain P53 and Mdm2 proteins if the Mdm2 induced delay $\tau_{1}$ in degradation of plain P53 is greater than the threshold $\tau_{1,0}$ and large (Theorem 4.1). In case, when the system is stressed $(s=1)$, our analysis uses Nyquist criterion to determine thresholds $\tau_{1}^{*}$ and $\tau_{2}^{*}$ under different conditions on model parameters such that delays $\tau_{1}$ and $\tau_{2}$ satisfying either $\max \left(\tau_{1}, \tau_{2}\right)=\tau<\tau_{1}^{*}$ or $\tau<\tau_{2}^{*}$ guarantee stability of the system. The oscillatory behavior in plain P53, active $\mathrm{P} 53$ and $\mathrm{Mdm} 2$ proteins if any, may be observed beyond these thresholds (Theorem 4.2). Since the comparative study of this paper with Bottani and Grammaticos model through numerical simulations for specific set of parameter values shows difference in the dynamics of plain P53, active P53 and Mdm2 proteins specially in the early stages or in terms of peaks of oscillations, we suggest that more laboratory experiments may be undertaken to corroborate the results of this paper especially during the initial and the advanced stages of cancers.

\section{References}

[1] Y. Haupt, R Maya, A. Kazaz and M. Oren, Mdm2 promotes the rapid degradation of p53, Nature 387(1997), 296-299.

[2] B. Vogelstein, D. Lane and A. J. Levine, Surfing the p53 network, Nature 408(2000), 307-310.

[3] W. P. Bennett, S. P. Hussain, K. H. Vahakangas, M. A. Khan, P. G. Shields and C. C. Harris, Molecular epidemiology of human cancer risk: gene-environment interactions and p53 mutation spectrum in human lung cancer. The Journal of pathology 187(1999), 8-18.

[4] Y. Barak, T. Juven, R. Haffner and M. Oren, $\mathrm{mdm} 2$ expression is induced by wild type p53 activity, The EMBO journal 12(1993), 461-468.

[5] I. L. Hayon and Y. Haupt, p53: an internal investigation, Cell Cycle 1(2002), 105-110. 
[6] D. Michael and M. Oren, The p53-Mdm2 module and the ubiquitin system, Seminars in Cancer Biology 13(2003), 49-58.

[7] J. Momand, H. H. Wu and G. Dasgupta, MDM2-master regulator of the p53 tumor suppressor protein. Gene 242(2000), 15-29.

[8] F. Toledoa and G. M. Wahl, Regulating the p53 pathway: in vitro hypotheses, in vivo veritas. Nature Reviews Cancer 12(2006), 909-923.

[9] M. UM and O. Petrenko, The MDM2-p53 interaction. Mol. Cancer Res 1(2003), 1001-1008.

[10] J. J. Tyson, (2002) Biochemical Oscillations. In: C. P. Fall, E. S. Marland, J. M. Wagner, J. J. Tyson, (eds) Computational Cell Biology. Interdisciplinary Applied Mathematics, Vol. 20. Springer, New York, NY

[11] A. Ciliberto, B. Novák and J. J. Tyson, Steady states and oscillations in the p53/Mdm2 network. Cell Cycle 4(2005), e107-e112.

[12] D. A. Hamstra, M. S. Bhojani, L. B. Griffin, B. Laxman, B. D. Ross and A. Rehemtulla, Realtime evaluation of p53 oscillatory behavior in vivo using bioluminescent imaging, Cancer Research 66(2006), 7482-7489.

[13] G. Lahav, N. Rosenfeld, A. Sigal, N. Geva-Zatorsky, A. J. Levine, M. B. Elowitz and U. Alon, Dynamics of the p53-Mdm2 feedback loop in individual cells, Nature Genetics 36(2004), $147-150$.

[14] N. G. Zatorsky, N. Rosenfeld, S. Itzkovitz, R. Milo, A. Sigal, E. Dekel, T. Yarnitzky, Y. Liron, P. Polak, G. Lahav and U. Alon, Oscillations and variability in the p53 system. Molecular Systems Biology 2(2006), 1-13.

[15] T. Zhang, P. Brazhnik and J. J. Tyson, Exploring mechanisms of the DNA-damage response: p53 pulses and their possible relevance to apoptosis. Cell Cycle 6(2007), 5-94.

[16] R. L. Bar-Or, R. Maya, L. A. Segel, U. Alon, A. J. Levine and M. Oren, Generation of oscillations by the $\mathrm{p} 53-\mathrm{Mdm} 2$ feedback loop: a theoretical and experimental study. Proceedings of the National Academy of Sciences 97(2000), 11250-11255.

[17] D. Brewer, Investigations of the $\mathrm{p} 53$ protein DNA damage network using mathematical models. University College London, London (UK). 2002 Oct.

[18] E. Batchelor, C. S. Mock, I. Bhan, A. Loewer and G. Lahav, Recurrent initiation: a mechanism for triggering p53 pulses in response to DNA damage. Molecular Cell 30(2008), 277-289. 
[19] E. Batchelor, A. Loewer, C. Mock and G. Lahav, Stimulus-dependent dynamics of p53 in single cells. Molecular Systems Biology 7(2011), 1-8.

[20] J. D. Murray, editor. Mathematical Biology I. An Introduction, third edn. Springer, 2002.

[21] T. F. Thingstad and T. I. Langeland, Dynamics of chemostat culture: The effect of a delay in cell response. Journal of Theoretical Biology 48(1974), 149-159.

[22] J. D. Murray, editor. Mathematical Biology II. Spatial Models and Biomedical Applications. Springer, 2003.

[23] G. I. Mihalas, Z. Simon, G. Balea and E. Popa, Possible oscillatory behavior in p53-Mdm2 interaction computer simulation. Journal of Biological Systems 8(2000), 21-29.

[24] G. I. Mihalaş, M. Neamțu, S. D. Opriş and R. F. Horhat, A dynamic P53-MDM2 model with time delay. Chaos, Solitons and Fractals 30(2006), 936-945.

[25] S. Bottani and B. Grammaticos, Analysis of a minimal model for p53 oscillations. Journal of Theoretical Biology 249(2007), 235-245.

[26] R. K. Layek, A. Datta and E. R. Dougherty, From biological pathways to regulatory networks. Molecular BioSystems 7(2011), 843-851.

[27] J. E. Purvis, K. W. Karhohs, C. Mock, E. Batchelor, A. Loewer and G. Lahav, p53 dynamics control cell fate. Science 336(2012), 1440-1444.

[28] T. Sun, W. Yang, J. Liu and P. Shen, Modeling the basal dynamics of p53 system. PloS One 6(2011), 1-9.

[29] K. H. Chong, S. Samarasinghe and D. Kulasiri, Mathematical modelling of p53 basal dynamics and DNA damage response. Mathematical Biosciences 259(2015), 27-42.

[30] V. Pant and G. Lozano, Dissecting the p53-Mdm2 feedback loop in vivo: uncoupling the role in p53 stability and activity. Oncotarget 5(2014), 1149-1156.

[31] L. Pecorino, Molecular biology of cancer: mechanisms, targets, and therapeutics. Oxford university press, 2012.

[32] X. Yang, L. Chen and J. Chen, Permanence and positive periodic solution for the singlespecies nonautonomous delay diffusive models. Computers and Mathematics with Applications 32(1996), 109-116.

[33] Y. Kuang, editor. Delay differential equations: with applications in population dynamics. Academic Press, 1993. 
[34] H. I. Freedman and V. S. Rao, Stability criteria for a system involving two time delays. SIAM Journal on Applied Mathematics 46(1986), 552-560.

Mohd Younus Baba Department of Applied Mathematics Aligarh Muslim University Aligarh

E-mail: younusmaths@gmail.com

Mohammad Saleem Professor (Retd) Department of Applied Mathematics Aligarh Muslim University Aligarh

E-mail: saleemmd60@gmail.com

Abdur Raheem Department of Mathematics Aligarh Muslim University Aligarh

E-mail: araheem.iitk3239@gmail.com 